4

\title{
Novel fluorochromes label tonoplast in living plant cells and reveal changes in vacuolar organization after treatment with protein phosphatase inhibitors
}

\author{
Miklós Nagy ${ }^{1}$ - Sándor Kéki ${ }^{1}$ - Dávid Rácz ${ }^{1}$. Jaideep Mathur ${ }^{2}$ - György Vereb ${ }^{3}$ - Tamás Garda ${ }^{4}$ Márta M-Hamvas ${ }^{4}$. \\ François Chaumont ${ }^{5} \cdot$ Károly Bóka $^{6,7} \cdot$ Béla Böddi $^{6,7} \cdot$ Csongor Freytag $^{4} \cdot$ Gábor Vasas $^{4} \cdot$ Csaba Máthé $^{4}$
}

Received: 23 May 2017 / Accepted: 27 November 2017

(C) Springer-Verlag GmbH Austria, part of Springer Nature 2017

\begin{abstract}
The recently synthesized isocyanonaphtalene derivatives ACAIN and CACAIN are fluorochromes excitable at wavelengths of around $366 \mathrm{~nm}$ and bind cysteine-rich proteins with hydrophobic motifs. We show that these compounds preferentially label tonoplasts in living Arabidopsis and tobacco (Nicotiana tabacum SR1) cells. ACAIN-labeled membranes co-localized with the GFP signal in plants expressing GFP- $\delta$-TIP (TIP2;1) (a tonoplast aquaporin) fusion protein. ACAIN preserved the dynamics of vacuolar structures. tip2;1 and triple tip1;1-tip1;2-tip2;1 knockout mutants showed weaker ACAIN signal in tonoplasts. The fluorochrome is also suitable for the labeling and detection of specific (cysteine-rich, hydrophobic) proteins from crude cell protein extracts following SDS-PAGE and TIP mutants show altered labeling patterns; however, it appears that ACAIN labels a large variety of tonoplast proteins. ACAIN/ CACAIN could be used for the detection of altered vacuolar organization induced by the heptapeptide natural toxin microcystin-LR (MCY-LR), a potent inhibitor of both type 1 and $2 \mathrm{~A}$ protein phosphatases and a ROS inducer. As revealed both in plants with GFP-TIP2;1 fusions and in wild-type (Columbia) plants labeled with ACAIN/CACAIN, MCY-LR induces the formation of small vesicles, concomitantly with the absence of the large vegetative vacuoles characteristic for differentiated cells. TEM studies of MCY-LR-treated Arabidopsis cells proved the presence of multimembrane vesicles, with characteristics of lytic vacuoles or autophagosomes. Moreover, MCY-LR is a stronger inducer of small vesicle formation than okadaic acid (which inhibits preferentially PP2A) and tautomycin (which inhibits preferentially PP1). ACAIN and CACAIN emerge as useful novel tools to study plant vacuole biogenesis and programmed cell death.
\end{abstract}

Keywords ACAIN/CACAIN · Arabidopsis $\cdot$ Tonoplast $\cdot$ Hypocotyl $\cdot$ Microcystin-LR $\cdot$ Vacuolar organization

Handling Editor: Liwen Jiang

Electronic supplementary material The online version of this article (https://doi.org/10.1007/s00709-017-1190-0) contains supplementary material, which is available to authorized users.

Csaba Máthé

mathe.csaba@science.unideb.hu

1 Faculty of Science and Technology, Department of Applied Chemistry, University of Debrecen, Egyetem tér 1, Debrecen H-4032, Hungary

2 Department of Molecular and Cellular Biology, Laboratory of Plant Development and Interactions, University of Guelph, 50 Stone Road, Guelph, ON N1G2W1, Canada
3 Faculty of Medicine, Department of Biophysics and Cell Biology, University of Debrecen, Debrecen, Hungary

4 Faculty of Science and Technology, Department of Botany, University of Debrecen, Debrecen, Hungary

5 Institut des Sciences de la Vie, Université catholique de Louvain, B-1348 Louvain-la-Neuve, Belgium

6 ELTE Eötvös Loránd University, Budapest, Hungary

7 Department of Plant Anatomy, Faculty of Science, Institute of Biology, Pázmány P. s. 1/c, Budapest H-1117, Hungary 


\section{Introduction}

Recently, two novel fluorochromes, 1-(2-acryloyloxy-3chloro-prop-1-yl)-amino-5-isocyanonaphthalene (ACAIN) and its chlorinated derivative (2-chloroacryloyloxy-3chloroprop-1-yl)-amino-5-isocyanonaphthalene (CACAIN) (Fig. 1), were synthesized in our laboratory. These are low MW molecules (315 Da for ACAIN) (Nagy et al. 2016). They have characteristic absorption spectra in the UV wavelength range with a peak at $366 \mathrm{~nm}$ for ACAIN, but absorption persists up to $405 \mathrm{~nm}$. They are excitable with emissions at the blue and green wavelength range when a stock dissolved in DMSO is diluted in water (peaks at 423 and $502 \mathrm{~nm}$ ) (Nagy et al. 2016). Moreover, ACAIN and its more reactive derivative CACAIN proved to bind lysil-alanyl-cysteine (KAC) as well as bovine serum albumin at hydrophobic motifs (Nagy et al. 2016). Due to the hydrophobic nature of these compounds, we suspected they have the potential to penetrate easily through biological membranes and label intracellular structures containing proteins with hydrophobic motifs.

Microcystin-LR (MCY-LR) is a natural cyclic heptapeptide toxin produced by several cyanobacterial genera. It is known to be a potent inhibitor of the serine-threonine protein phosphatases, mainly of type 1 and 2A (PP1 and PP2A), and of PP3, PP4, PP5, and PP6 (Swingle et al. 2007). It is also a strong inducer of reactive oxygen species (ROS) (Bouaïcha and Maatouk 2004; Campos and Vasconcelos 2010). Due to these two properties, it affects key cellular events: metabolic regulation, signal transduction events, and cell-cycle regulation. Thus, it affects eukaryotic subcellular structures: chromatin, cytoskeletal, and endomembrane (mainly ER) organization (Chen and Xie 2016; Máthé et al. 2013, 2016). For this reason, it is ideal for the study of alterations of subcellular organization under stress. If ACAIN and CACAIN can label specific subcellular structures, MCY-LR could be ideal to be

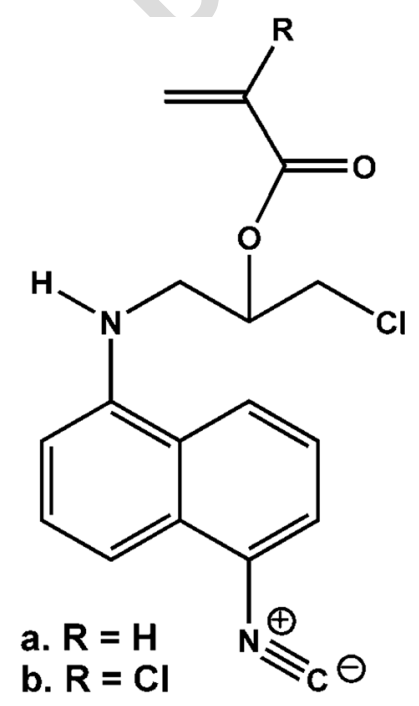

Fig. 1 Chemical formulae of ACAIN $(a, R=H)$ and CACAIN $(b, R=C l)$ used as a tool for the detection of changes highlighted by these fluorochromes.

In the light of the above arguments, our main goals were (1) to detect the subcellular structures labeled by ACAIN and CACAIN and to assess whether they can label proteins related to those subcellular structures, and (2) to test these fluorochromes for their suitability to detect stress-induced subcellular alterations induced by MCY-LR. We show that ACAIN/CACAIN are suitable as alternative labels for specific cellular structures-tonoplasts - and for detecting changes in the organization of tonoplast-coated endomembranes.

\section{Materials and methods}

\section{Chemicals}

ACAIN/CACAIN were synthesized as we have previously described (Nagy et al. 2016). Briefly, the synthesis started from 1,5-diaminonaphthalene (Sigma-Aldrich, St. Louis, MO, USA) by Hofmann isonitrile synthesis using chloroform and potassium hydroxide, followed by the addition of epichlorohydrin on the amine group. Secondary hydroxyl group formed was then acylated with acryloyl chloride (ACAIN) or 2-choloroacrylic acid (CACAIN). MCY-LR was purified from Microcystis aeruginosa BGSD243 according to the procedure of Kós et al. (1995) modified by Vasas et al. (2004). Briefly, methanolic extracts of $M$. aeruginosa cells were vacuum evaporated, re-extracted with $10 \mathrm{mM}$ Tris- $\mathrm{HCl}, \mathrm{pH} 7.5$, and subjected to ion-exchange chromatography on DEAEcellulose (DE-52; Whatman, Maidstone, UK) columns. This was followed by desalting and semi-preparative HPLC to obtain MCY-LR of purity $\geq 95 \%$.

\section{Plant material and treatments}

Arabidopsis thaliana genotypes used were Col-0 (wild-type), plants bearing GFP-TIP2;1 ( $\delta$-TIP) fusion protein described by Cutler et al. (2000)), and four knockout mutants in Col-0 background of tonoplast intrinsic proteins (TIPs). These were tip 1;1, tip 1;2, tip2;1, and their triple mutant obtained as described previously (Reinhardt et al. 2016). The tobacco genotype used was Nicotiana tabacum SR1. Seeds were surface sterilized by rinsing two times for 10 min with $10 \%$ of a sodium-hypochlorite-containing solution, then washed four to five times for $5 \mathrm{~min}$ with sterile water. Seeds were transferred to Murashige-Skoog (MS) basal medium supplemented with Gamborg's vitamins, 2\% (w/v) sucrose (Molar, Budapest, Hungary), and 0.8\% (w/v) Bacto-agar (Difco, Lawrence, KS, USA) (Gamborg et al. 1968; Murashige and Skoog 1962). After a 48 -h cold treatment (used for Arabidopsis), plates were placed in a tissue culture room 
114 (14:10 h photoperiod, $22 \pm 2{ }^{\circ} \mathrm{C}, 60 \mu \mathrm{mol} \mathrm{m}{ }^{-2} \mathrm{~s}^{-1}$ photon flux 115 density in the light period). After 1 week of culture, part of the 116 seedlings was directly subjected to ACAIN/CACAIN cyto117 logical labeling or ACAIN staining of root and shoot extracts 118 after SDS-PAGE (see sections below). For detecting ACAIN119 labeled proteins on SDS-PAGE, Lemna minor shoots axeni120 cally subcultured for 1 week on liquid Allen medium (Allen 121 1968) were used as well. Part of Arabidopsis (Col-0 and GFP122 TIP2;1) seedlings were further treated with protein phospha123 tase inhibitors. Col-0 and GFP-TIP2;1 seedlings were treated 124 with 1-5 $\mu \mathrm{M}$ MCY-LR for 4-72 h. Col-0 plants were further 125 stained with ACAIN/CACAIN. GFP-TIP2;1 seedlings were 126 also treated with $1 \mu \mathrm{M}$ okadaic acid (OA) for $24 \mathrm{~h}$ and $1 \mu \mathrm{M}$ 127 tautomycin (Tm) for 4-24 h.

\section{ACAIN/CACAIN staining and microscopy}

Control and protein phosphatase-treated whole seedlings were placed in four-well tissue culture chambers for microscopy (Sarstedt, Nümbrecht, Germany) and washed with phosphate buffered saline (PBS). ACAIN/CACAIN stocks dissolved in DMSO were diluted in PBS with or without $0.05 \%(v / v)$ Triton X-100 (Reanal, Budapest, Hungary) to obtain final concentrations of $20 \mu \mathrm{g} \mathrm{mL}$, used for labeling of seedlings. Duration of labeling was $30 \mathrm{~min}$, followed by three consecutive 5-min washes with PBS. Samples were then first visualized with a conventional Olympus Provis AX-70 (Olympus, Tokyo, Japan) fluorescence microscope at excitation wavelength of 320 $360 \mathrm{~nm}$. This was followed by CLSM analysis. ACAIN/ CACAIN stained Col-0 and GFP-TIP2;1 seedlings were analyzed with a Zeiss LSM 880 confocal microscope by using a $\times 40 \mathrm{C}$-Apochromat water immersion objective. Excitation and emission wavelengths were 405/408$473 \mathrm{~nm}$ (ACAIN/CACAIN), 488/492-540 nm (GFP), and 543/547-614 nm (chlorophyll autofluorescence). For treatment of GFP::TIP2;1 seedlings with protein phosphatase inhibitors (without ACAIN/CACAIN labeling), a Leica TCS-SP5 confocal microscope/HCX APO L U-V-I $40.0 \times 0.80$ water immersion objective was used. GFP was excited with a $488 \mathrm{~nm}$ Ar laser, and emission was at 503$515 \mathrm{~nm}$. For chlorophyll autofluorescence detection, the $543 \mathrm{HeNe}$ laser was used. Emission was at $650-710 \mathrm{~nm}$, and chlorophyll autofluorescence was pseudocolored in blue on the GFP-chlorophyll composite images.

All cytological experiments were performed at least three times, and representative results are presented ("Results" section).

\section{TEM studies}

Col-0 seedlings were treated with $1-2 \mu \mathrm{M}$ MCY-LR for $72 \mathrm{~h}$ (see above). TEM preparations were made as follows: segments of control and microcystin treated Arabidopsis thaliana hypocotyls were fixed in 2\% glutaraldehyde for $3 \mathrm{~h}$ and in $1 \%$ osmium tetroxide for $2 \mathrm{~h}$ (both fixatives were dissolved in $0.1 \mathrm{M} \mathrm{K}-\mathrm{Na}$-phosphate buffer, $\mathrm{pH}$ 7.2) rinsed in the buffer and embedded in Durcupan resin after dehydration in an ethanol series ended with propylene oxide. Ultra-thin sections $(70 \mathrm{~nm})$ were cut with a Reichert Ultracut E ultramicrotome (Leica Microsystems, Vienna, Austria), mounted on uncoated $\mathrm{Cu} / \mathrm{Pd}$ grids (Polysciences, Warrington, PA, USA), and stained with $2 \%$ uranyl acetate dissolved in methanol for $4 \mathrm{~min}$ and lead citrate for $6 \mathrm{~min}$. Sections were studied with a Hitachi 7100 transmission electron microscope (Hitachi, Tokyo, Japan) at $75 \mathrm{kV}$.

\section{ACAIN labeling of proteins following SDS-PAGE}

Protein extracts from shoots and roots of Arabidopsis and tobacco seedlings as well as Lemna minor shoots were prepared in the SDS (Sigma-Aldrich) containing standard Laemmli buffer (Laemmli 1970), but without boiling to allow further renaturation of proteins (see 2-propanol treatments below). To check whether this extraction does not allow protein degradation, alternatively extracts were prepared in the presence of a protease inhibitor cocktail $(0.5 \% \mathrm{v} / \mathrm{v}$; Roche Applied Science, Indianapolis, USA) as well as without protease inhibitors and with standard protein boiling. Protein contents of samples were assayed by the Bradford (1976) method. Extracts containing $40 \mu \mathrm{g}$ protein per well were loaded along with molecular weight markers (Sigma-Aldrich, BLUeye Prestained Protein Ladder). SDS-containing gels were prepared essentially by the Laemmli (1970) method except that 7.5-18\% polyacrylamide gradient (running) gels proved to be the best for our purposes and used accordingly. After SDSPAGE, gels were rinsed for $10 \mathrm{~min}$ in sterile water, then twice for $10 \mathrm{~min}$ in $20 \%(v / v)$ 2-propanol (VWR, Radnor, USA) and twice for $20 \mathrm{~min}$ in PBS. Afterwards, labeling was performed for $5 \mathrm{~h}$ with $35 \mu \mathrm{g} \mathrm{mL}^{-1}$ ACAIN in PBS. Gels were then washed for 10 min with 20\% 2-propanol, followed by two consecutive washes with PBS for 5 min each. ACAINlabeled proteins were detected by illumination at $365 \mathrm{~nm}$ and photographed with an UVITEC transilluminator/gel documentation equipment (UVITEC, Cambridge, UK). MW of proteins was determined with the UVITEC $®$ software. Band intensities and intensity curves were determined with the aid of the GelAnalyzer 2010 software. Besides ACAIN staining, gel slices containing the same protein samples were stained with $0.2 \%$ Coomassie Brilliant Blue (Serva, Heidelberg, Germany) for $4 \mathrm{~h}$.

All SDS-PAGE work was performed in three independent experiments and images of representative gels are shown in the "Results" section. 


\section{Results}

\section{ACAIN labels tonoplasts in different plant species 215 and TIPs contribute to this labeling pattern}

When living Col-0 plants were stained with ACAIN and visualized with a conventional fluorescence microscope, specialized shoot epidermal cells (trichomes and guard cells) as well as hypocotyl cortex cells showed endomembrane labeling. Labeling pattern raised the possibility of ACAIN binding to vacuolar membranes (Fig. 2a-d). Therefore, in the next step, we labeled whole Arabidopsis seedlings expressing GFP-TIP2;1, known for the characteristic tonoplast GFP signal (Cutler et al. 2000). ACAIN and GFP signals showed a perfect co-localization in hypocotyls (Fig. 2e). Labeling of Col- 0 and GFP-TIP2; 1 seedlings showed the presence of large vacuoles as well as smaller tonoplast-coated vesicles in hypocotyl epidermal and cortex cells (Fig. 2e, f).
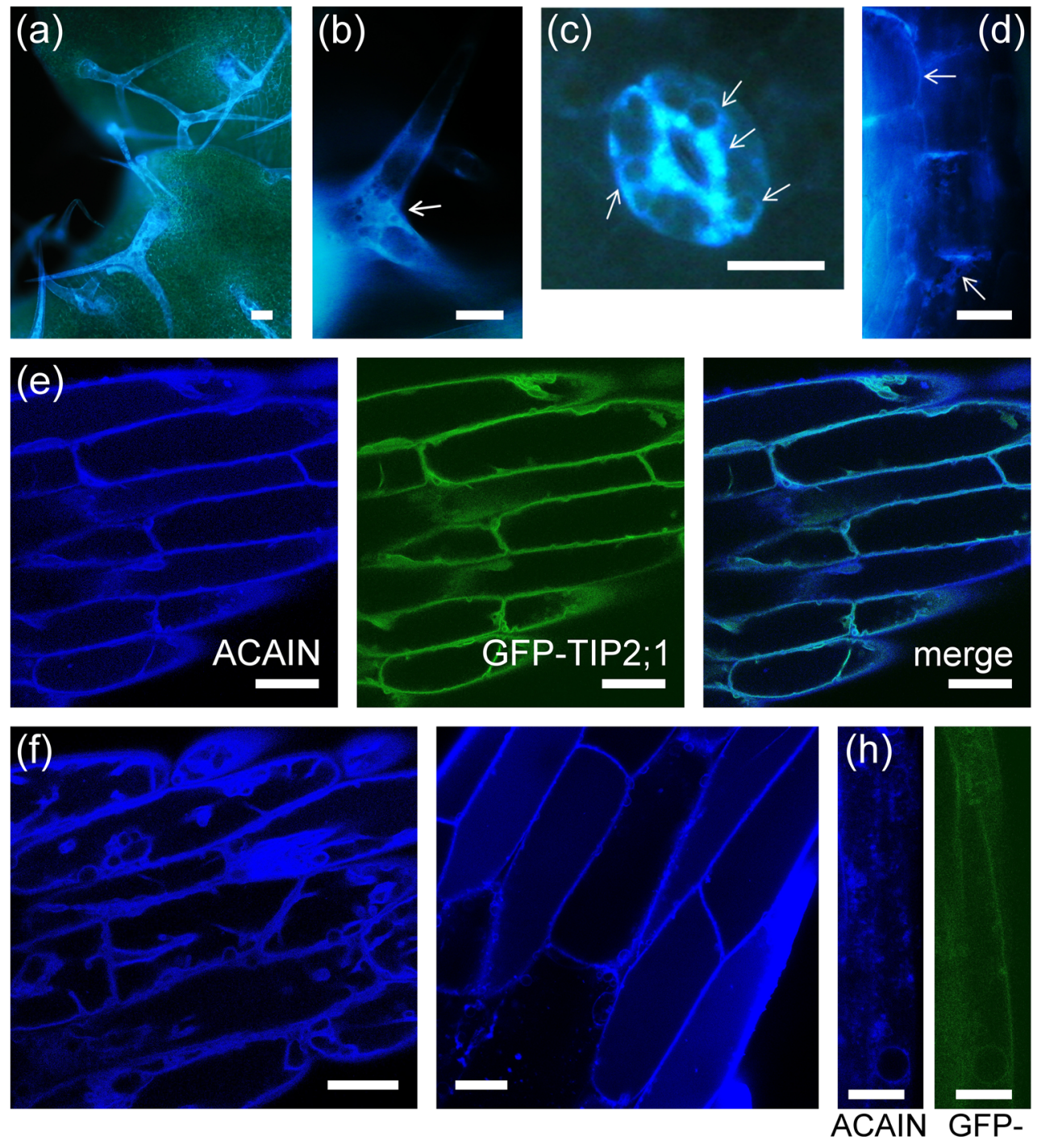

TIP2; 1

Fig. 2 ACAIN labels exclusively tonoplasts in living plant cells. All images were taken from Arabidopsis seedlings, except (g) that was of hypocotyls from $N$. tabacum SR1 seedlings. Images of (a)-(d) are of conventional fluorescence microscopy with arrows indicating the labeling of membrane structures. (e)-(h) are CLSM images. ACAIN labeling (a) of trichomes, (b) detail of trichome labeling, (c) of hypocotyl guard cells, and (d) of hypocotyl cortex cells from Col-0 plants. Conventional fluorescence microscope images show the probability of endomembrane labeling. e ACAIN labeling of a Triton
X-100 permeabilized hypocotyl segment of Arabidopsis Col plants containing GFP-tagged $\delta$-TIP (TIP2;1) protein. ACAIN label colocalizes with TIP2;1. f, g ACAIN labeling of hypocotyl cells not permeabilized with Triton X-100 from Arabidopsis Col-0 (f) and N. tabacum SR1 (g). h ACAIN labeling of Triton X-100 permeabilized differentiated cells of root from Arabidopsis plants containing GFPtagged $\delta$-TIP (TIP2;1) protein. Both ACAIN and GFP signals are diffuse, showing multiple tonoplast-coated vesicles. Scale bars: $30 \mu \mathrm{m}$ 
To exclude the possibility that ACAIN labels only tonoplasts because it cannot penetrate into other subcellular structures, ACAIN labeling was performed in the presence of increased DMSO concentration (10\%) and/or $0.05 \%$ Triton X-100 as well, but these well-known compounds that increase membrane permeability did not change the labeling pattern (compare Fig. 2e and f). ACAIN could label tonoplasts in hypocotyl cells of tobacco ( $N$. tabacum SR1) as well, in a similar manner to Arabidopsis (Fig. 2f, g). The fluorochrome preserved cell viability and vacuolar dynamics, thus it proved to be suitable for live cell imaging (Supplementary Movie 1). For differentiated or meristematic cells of Arabidopsis roots, labeling was weaker and more diffuse, but this was true for the GFP signal in GFP-TIP2; 1 roots as well (Fig. 2h). Concerning specificity of ACAIN for tonoplast, other endomembrane structures like the ER or Golgi apparatus had fluorescent signals clearly different to ACAIN labeling in transgenic Arabidopsis plants with GFP- or YFP-fusion proteins specific for those organelles (Supplementary Fig. 1a-c). Plasmolyzed hypocotyl cells of YFP-PIP2a plants (with YFP signal of the plasma membrane) showed nearly exclusive YFP label of Hechtian strands and exclusive ACAIN label of shrunk vacuoles (Supplementary Fig. 1d, e). Plants with ER/Golgi/plasma membrane YFP/GFP signals were from Boevink et al. (1998), Cutler et al. (2000), Mathur et al. (2003), and Nelson et al. (2007)).

Different TIP knockout mutants showed altered ACAIN labeling in hypocotyls as compared to Col-0 plants. tip1;1 and tip 1;2 mutants showed weaker, but still well-detectable tonoplast labeling (compare Fig. 3a-c). For tip 2;1 and the triple tip1;1-tip1;2-tip2;1 mutants, ACAIN signal was not detectable at excitation settings identical to Col-0 seedlings, but brightness-contrast adjustment showed that it still labeled tonoplasts, although the signal was very weak (Fig. 3d, d', e, e').

\section{ACAIN labels proteins on SDS-polyacrylamide gels}

The extraction of proteins in Laemmli buffer - due to its SDS content (see "Materials and methods" section)_allowed obtaining of crude cellular protein extracts that contained membrane proteins. For Col-0 seedlings, shoot extracts contained several proteins that were labeled by ACAIN: the most characteristic bands were proteins of 27 (band 1), 24 (band 2), 19, and $18 \mathrm{kDa}$ (double band $3 \mathrm{a}$ and $3 \mathrm{~b}$ ) (see arrows on Fig. 4a). Small (below $11 \mathrm{kDa})$ and larger $(\geq 35 \mathrm{kDa})$ MW proteins could also be detected (Fig. 4a). We could not detect ACAIN-labeled proteins in Col-0 root extracts (Fig. 4a). Concerning shoot extracts of TIP mutants, the general labeling pattern was weaker in tip 1;1 and tip 2;1 as compared to controls (Fig. 4a). Shoot extracts of N. tabacum SR1 and L. minor contained ACAIN-labeled proteins as well. The most characteristic band had a MW of $24 \mathrm{kDa}$ for both extracts (Fig. 4b, arrow) and several other proteins showed weak labeling (Fig. 4b). To check whether the protein extraction method without boiling (see "Materials and methods" section) does protect
Fig. 3 ACAIN labeling in the absence of Triton X-100 in hypocotyls of TIP knockout mutants reveals weaker, but still existent tonoplast signal. Images of $(\mathbf{a}-\mathbf{d}, \mathbf{e})$ were taken with the same gain and laser intensity settings. a Col-0, ACAIN labeling, and chloroplast autofluorescence; b tip 1; 1; c tip1;2; d tip 2; 1, ACAIN labeling and chloroplast autofluorescence. $\left(\mathbf{d}^{\prime}\right)$ is the brightness/contrast adjusted version of (d) to show that the ACAIN signal is still present. e ACAIN labeling and chloroplast autofluorescence in the triple TIP knockout mutant. $\left(\mathbf{e}^{\prime}\right)$ is the brightness/contrast adjusted version of (e) to show that the ACAIN signal is still present. Scale bars: $30 \mu \mathrm{m}$
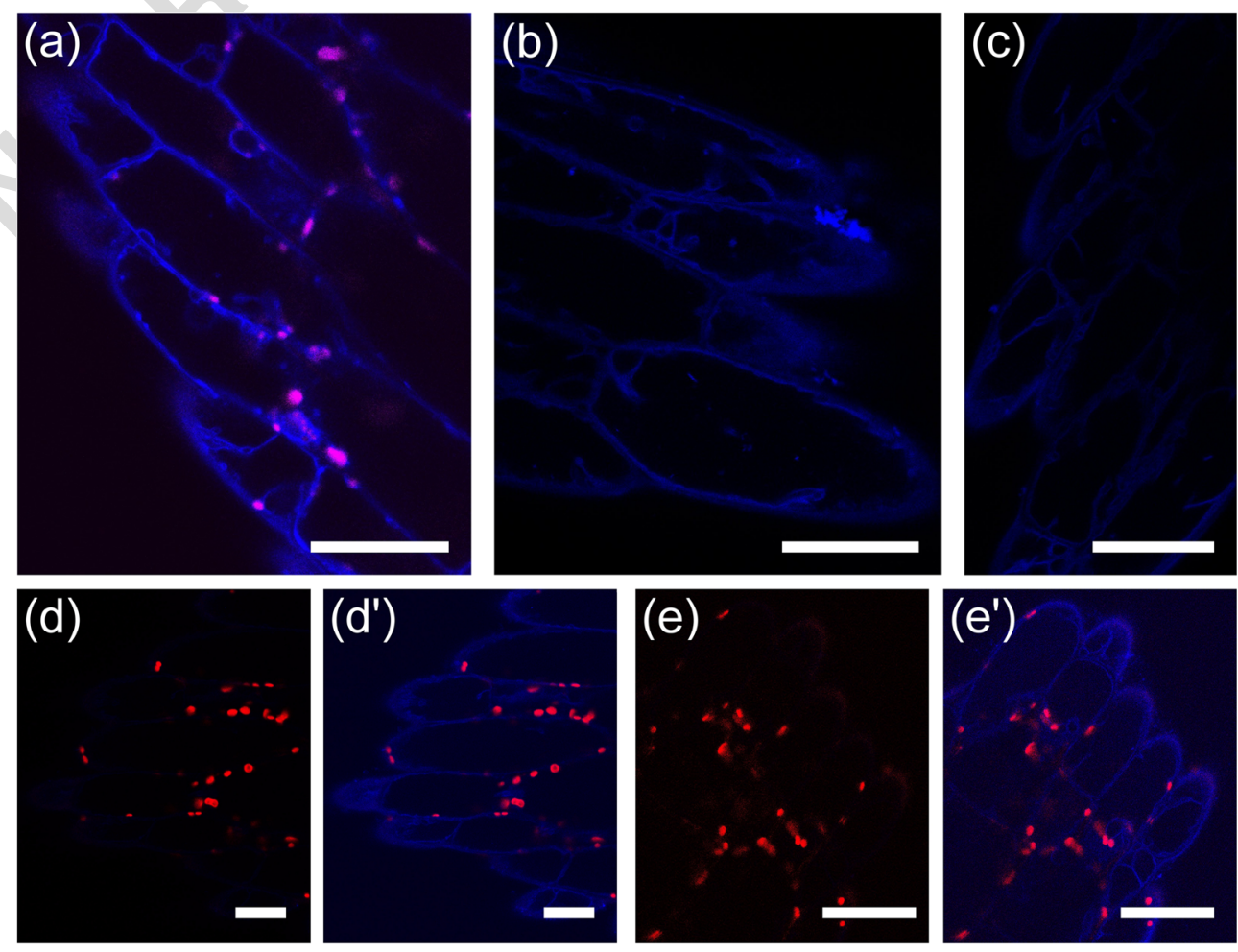


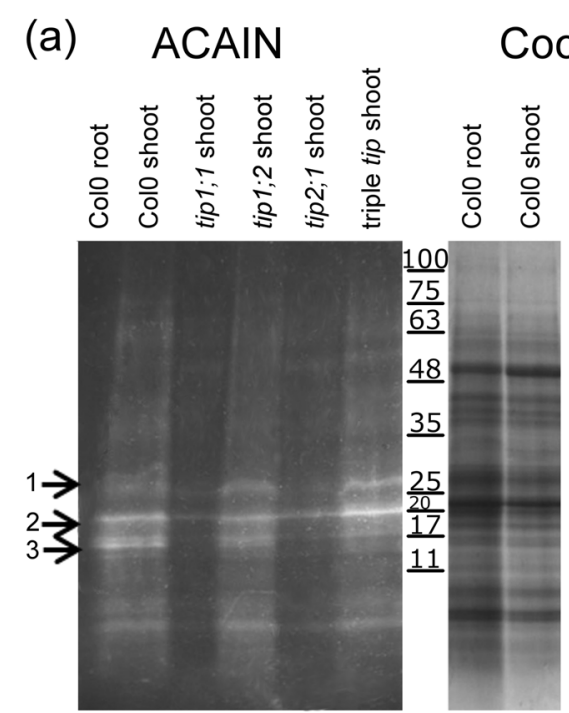

Fig. 4 ACAIN labels seedling proteins as revealed by SDS-PAGE. a ACAIN label of Arabidopsis (Col-0) roots and shoots (no labeling of roots), of shoots from Arabidopsis TIP mutants, and the corresponding total protein patterns (Coomasie Blue staining) along with a molecular weight marker. Arrows indicate the most important changes in the intensity of protein bands from mutants as compared to the wild-type

extracts from being degraded by proteases, we analyzed Col-0 extracts prepared in the presence of a protease inhibitor cocktail as well as boiled extracts. We could see no difference in protein patterns as compared to the samples prepared without boiling and protease inhibitors (Supplementary Fig. 2). This statement is valid for tobacco and duckweed extracts as well (data not shown).

Detailed analysis of intensity curves of SDS gels with Arabidopsis ACAIN-labeled protein bands showed the following changes in protein band patterns (Fig. 5): (1) for the 27- and 24-kDa proteins (bands 1-2), intensity in tip 1;1 and tip2;1 shoots decreased as compared to Col-0 shoots, while they remained unchanged in tip $1 ; 2$ shoots and increased in the triple TIP mutant (see arrows 1-2 on Fig. 4a). (2) for the 1819-kDa proteins (double band $3 \mathrm{a}$ and $3 \mathrm{~b}$ ), the presence of two proteins with similar MWs was clearly visible in Col-0 shoots (see inset box for Col-0 on Fig. 5). This double band nearly disappeared in tip 1;1 extracts and its intensity decreased in general in mutants. Moreover, one band was apparently missing in tip2;1 and triple TIP mutants (arrow 3 on Fig. $4 \mathrm{a}$ and inset box for triple mutant on Fig. 5).

\section{ACAIN/CACAIN labeling reveals changes in vacuolar organization induced by protein phosphatase inhibitors}

ACAIN and CACAIN staining gave identical labeling patterns of tonoplasts in Col-0 hypocotyls (Fig. 6a, b). CACAIN labeling of MCY-LR treated hypocotyls for relatively short term $(24 \mathrm{~h})$ showed the absence of large vacuoles in many cortex cells. Instead, small, tonoplast-coated vesicles were present (Fig. 6c). Many of these vesicles engulfed chloroplasts (Fig. 6c, arrows). However, engulfment of plastids by smaller tonoplast-coated vesicles could be observed in some control cells as well (data not shown). ACAIN labeling of MCY-LR-treated cells showed similar changes (data not shown).

GFP signal in hypocotyls from GFP-TIP2;1 seedlings treated with protein phosphatase inhibitors showed the following: controls showed normal vacuolar system (Fig. 6d). Four hours of MCY-LR treatment revealed the absence of large vacuoles and the presence of small tonoplast-coated vesicles similarly to the pattern revealed by CACAIN/ACAIN staining (Fig. 6e). Moreover, the movement/dynamics of these vesicles was very slow (Supplementary Movie 2). OA treatments did not alter vacuolar organization (Fig. 6f). In contrast, in the presence of $\mathrm{Tm}$, the proportion of large vacuoles decreased in many cells (while for MCY-LR, large vacuoles were completely lacking in several cells), and this effect increased over time ( 9 vs. $24 \mathrm{~h}$ ) (Fig. 6g-i). Thus, Tm did not have such dramatic effects on vacuolar organization as MCY-LR.

TEM studies showed normal organization of endomembranes in control Col-0 plants, with large vacuoles and smaller, abundant vesicles (Fig. 6j). After $72 \mathrm{~h}$ of treatment with 1-2 $\mu \mathrm{M}$ MCY-LR, an increase in the abundance of multimembrane vesicles as well as autophagosome-like structures incorporated into the large vacuoles were observed (Fig. 6k; Supplementary Fig. 3). Apparently, multimembrane vesicles formed in the cytosol (Fig. 6k) were later incorporated in the large vacuoles (Supplementary Fig. 3a). 

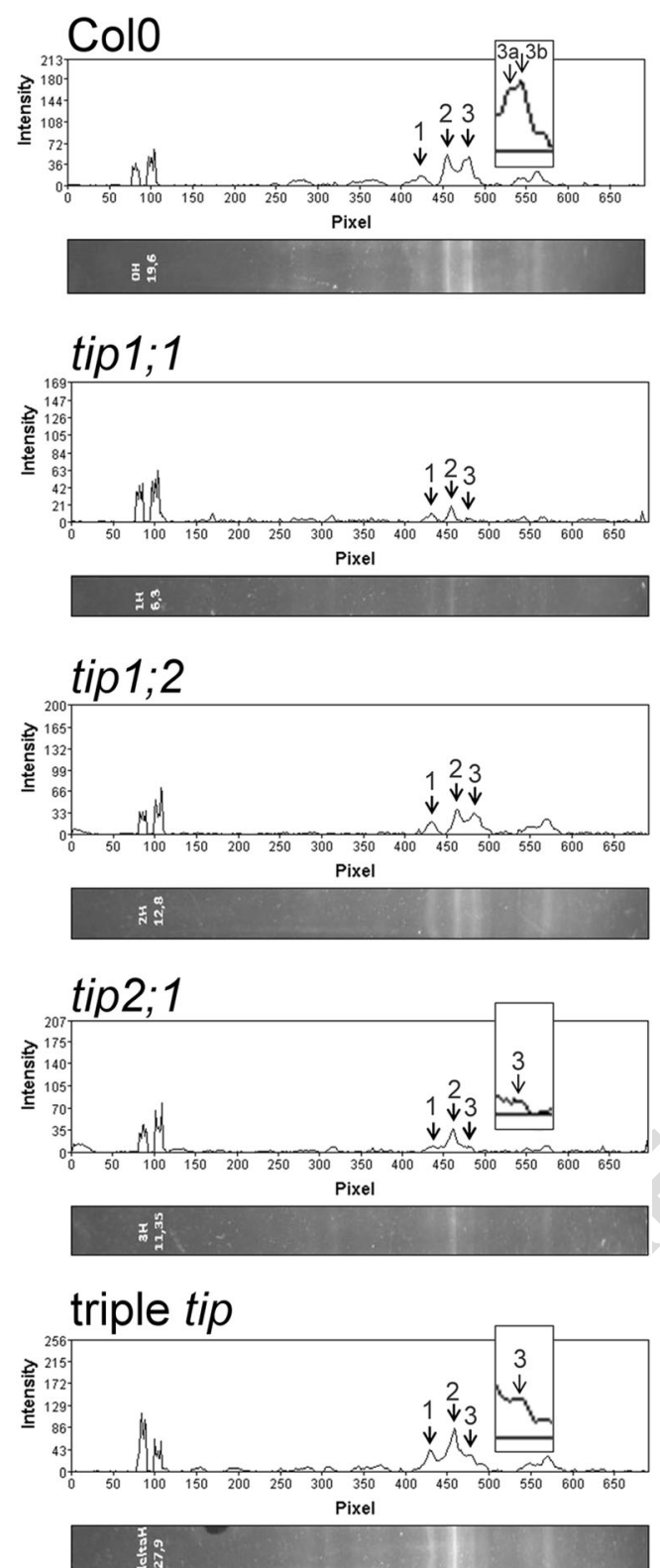

Fig. 5 Intensity curves of Arabidopsis wild-type and TIP mutant shoot protein patterns upon ACAIN labeling; analysis of the gel presented in Fig. 4a. Arrows show the intensity of characteristic bands indicated on Fig. 4a. Band of the $27-\mathrm{kDa}$ protein (arrow 1) shows relatively low intensity except the triple TIP mutant, where its labeling intensity increases. Band of the 24-kDa protein (arrow 2) shows decreased intensity for tip 1;1, tip1;2 and tip2;1 relative to Col-0 and increased intensity in the triple TIP mutant. The double band of $18-19-\mathrm{kDa}$ proteins (arrow 3 ) shows relatively high intensity for the Col-0 shoots (see inset box for the presence of two bands). All mutants show decreased intensity of labeling of this band, with its near absence in tip1;1. For tip2;1 and the double TIP mutant, apparently there is only one band (see inset boxes)

\section{Discussion}

ACAIN and CACAIN label preferentially tonoplasts in living manner (Figs. 2f, g and 6a, b). Tonoplast labeling is proven by the co-localization of fluorochrome signals with GFP signals from Arabidopsis plants expressing GFP-TIP2;1 fusion protein (Fig. 2e) reported to highlight all endomembranes delimited by a tonoplast (Cutler et al. 2000). The second evidence for preferential tonoplast labeling is that Hechtian strands known to consist mainly of plasma membrane fractions that tend to attach to the cell wall are showing almost exclusively the YFP label when plasmolyzed hypocotyl cells of YFP-PIP2a plants (Cutler et al. 2000) are labeled with ACAIN (Supplementary Fig. 1d, e). Meanwhile, shrunk vacuoles of plasmolyzed cells probably delimited only by tonoplasts are labeled exclusively by ACAIN (Supplementary Fig. 1d, e). The surface of shrunken protoplasm is apparently showing both the ACAIN and YFP label (Supplementary Fig. 1e). However, one should note that during plasmolysis, tonoplast or segments of tonoplast may be tightly adhered to the plasma membrane (Oparka 1994). While the resolution of light microscopy/CLSM might not allow us to distinguish plasma membrane and tonoplast under these conditions, the possible tight adherence of the two membrane types may lead one to false co-localization. The third evidence is the different fluorescence signal pattern (as compared to ACAIN/CACAIN) for other endomembranes (ER, Golgi) in Arabidopsis plants with GFP/YFP-fusion proteins labeling those cell compartments (Supplementary Fig. 1a-c). The fourth evidence for the peculiar ACAIN/CACAIN labeling pattern is that the use of known surfactants (Triton X-100) neither increased the intensity of labeling nor caused labeling of other subcellular structures than tonoplasts (Fig. 2e-g). Data presented in the "Results" section suggest that the isocyanonaphthalene derivatives used in this study can be of broad use for tonoplast labeling in living plant cells. Since it works well in another model plant too (N. tabacum SR1), ACAIN/CACAIN labeling could be a good alternative to plants expressing GFP-fusion proteins for cytological studies on the tonoplast. It can be used for species where transgenic lines with fluorescent protein labels of tonoplasts are not available.

What could be the nature of proteins labeled by our isocyanonaphthalene derivatives? Theoretically, a large variety of proteins containing hydrophobic motifs must be labeled by ACAIN and its derivatives (Nagy et al. 2016). Among plant vacuolar membrane proteins, tonoplast intrinsic proteins (TIPs) - since they are aquaporins - are fitting, although not exclusively into this category. Indeed, two Arabidopsis knockout mutants (tip2;1 and tip1;1-tip1;2-tip2;1 triple mutant) showed much weaker signal than the wild-type (Col-0) plants as revealed by CLSM (Fig. 2). Thus, at least some of the TIPs are labeled by ACAIN (and CACAIN), but not exclusively TIPs are labeled. Tonoplasts are characterized by a large variety of channel proteins characterized by hydrophobic transmembrane domains (Batistič 2012; Maeshima 2001). Indeed, ACAIN staining of protein gels revealed a large number of 

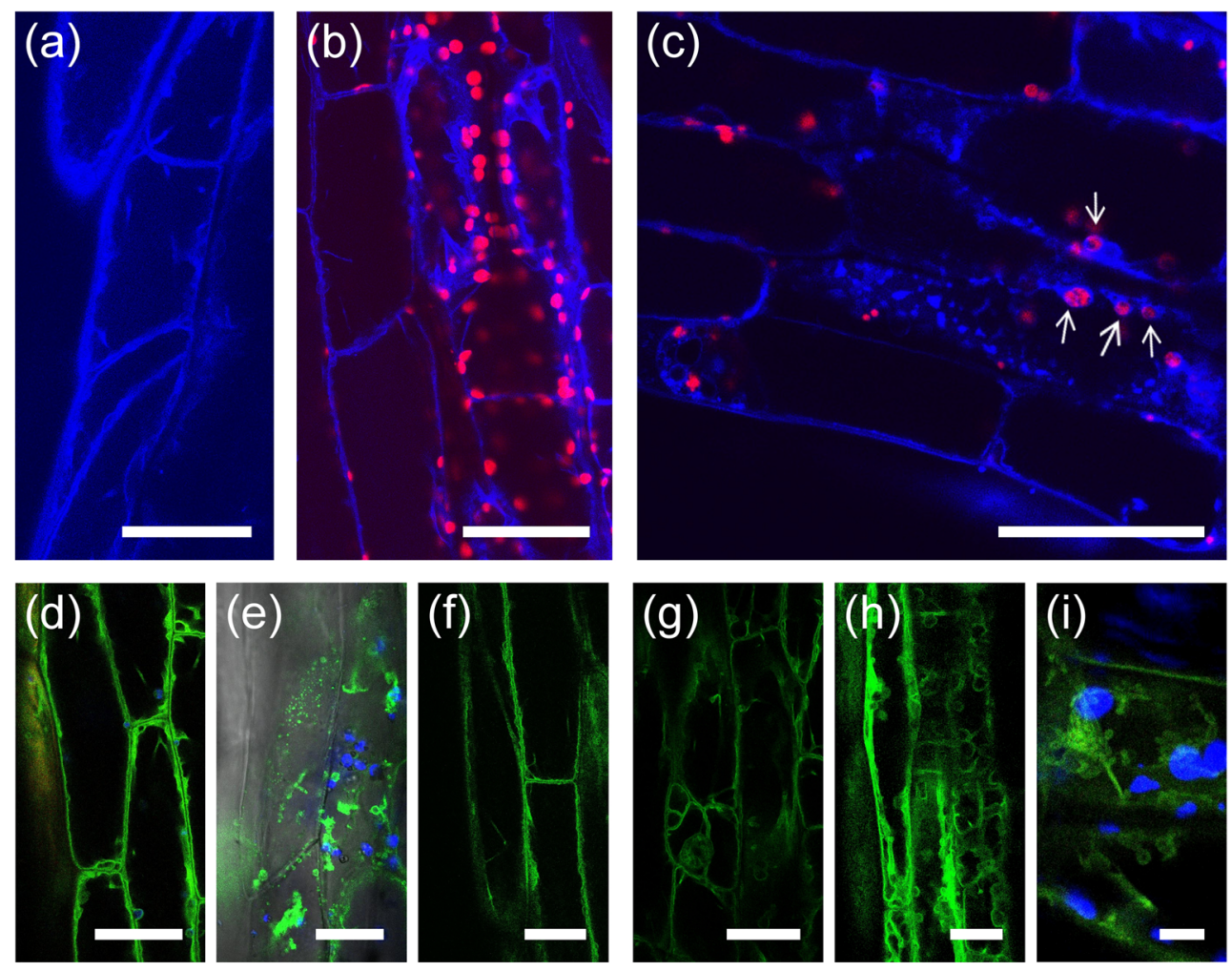
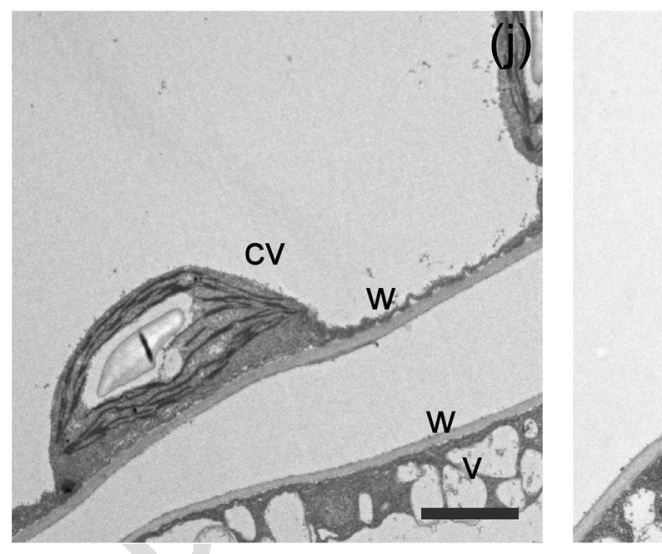

Fig. 6 CACAIN labeling in Col-0 (a-c) and analysis of GFP-TIP2; 1 (di) hypocotyls reveal changes of vacuolar organization in hypocotyl cells treated with protein phosphatase inhibitors. a Control, ACAIN labeling, normal vacuolar system; b control, CACAIN labeling, tonoplasts (blue) and chloroplast autofluorescence (red); c treatment with $5 \mu \mathrm{M}$ microcystin-LR (MCY-LR) for $24 \mathrm{~h}$, CACAIN labeling. Disorganization of the vacuolar system and capturing of chloroplasts by tonoplast-coated vesicles (arrows). d Control, normal vacuolar system; e $1 \mu \mathrm{M}$ MCY-LR, $4 \mathrm{~h}$ treatment showing numerous tonoplast-coated vesicles; $\mathbf{f} 1 \mu \mathrm{M}$ okadaic acid (OA), $24 \mathrm{~h}-$ no visible changes in the

labeled proteins. Among them, the 22-26-kDa TIPs (see
Maeshima 2001 and Schüssler et al. 2008 for molecular weights) were presumably labeled, but several other proteins were also well detectable (Fig. 4). Interestingly, the intensity of ACAIN labeling decreased for the 22-26-kDa proteins in the tip1;1 and tip2;1 mutants, but increased in the triple knockout mutant (Figs. 3 and 4), predicting that for the latter mutant, the absence of three TIP proteins is compensated by increased expression of other proteins. It should be noted, however, that in roots, in general the absence of specific TIPs in the mutants is not compensated by other TIP isoforms (Reinhardt et al. 2016). Thus, our prediction might be valid only for shoot extracts, where ACAIN labeling of proteins following SDS-PAGE was clearly visible (Fig. 4) or for other 
411 proteins than TIPs. It should be noted that to date, 10 proteins 412 of the TIP family were detected in Arabidopsis (the expression 413 of several TIPs is organ and tissue specific) and many other 414 proteins with hydrophobic motifs are present in the tonoplast 415 (Beebo et al. 2009). One of the most characteristic changes 416 revealed by SDS-PAGE of the proteins from wild-type plants 417 and mutants were the 18-19-kDa proteins (according to their 418 MW, probably not TIPs) that were almost completely absent 419 in the triple mutant (Figs. 4 and 5, "Results" section). At this 420 stage, we can assume that our fluorochromes are suitable for 421 the detection of specific proteins, among them tonoplast pro422 teins in a crude cellular extract.
(543 nm), and/or red (633 nm) wavelength excitation are used simultaneously.

In the next step, we were looking for the possible use of ACAIN/CACAIN for the study of stress-induced changes of vacuolar organization. We used microcystin-LR (MCY-LR) for this purpose (see "Introduction" section for reasons). This toxin is widely used as a tool for the regulation of structural organization and dynamics of subcellular compartments (Máthé et al. 2016). Vacuolar organization including the formation of lytic vacuoles and autophagosomes is sensitive to biotic and abiotic stresses including oxidative stress (Liu and Bassham 2012). ACAIN/CACAIN labeling revealed that MCY-LR, known to be a potent inhibitor of serine-threonine protein phosphatases (mainly PP1 and PP2A) and a ROS inducer, induced the formation of small tonoplast-coated vesicles, several of which engulfed chloroplasts (Fig. 6c). At this stage, we cannot state unequivocally the nature of these vesicles. Our TEM studies revealed that MCY-LR induced the formation of multimembrane vesicles as well as vesicles incorporated into the large vacuoles resembling plant autophagosome morphology (Fig. 6k, Supplementary Fig. 3) as described by Gao et al. (2015) and Liu et al. (2012). Autophagosomes can be not only double membrane but multimembrane vesicles as well. The multimembrane structures (Fig. 6k) were similar to autophagosomes in pathogeninfected Nicotiana benthamiana cells, as described by Liu et al. (2005). Autophagosomes are known to fuse with vacuoles, where their contents are degraded (Liu and Bassham 2012). The formation of multimembrane vesicles detected by our TEM studies could be the result of altered lytic vacuole formation in Arabidopsis as well (Feraru et al. 2010).

If the multimembrane vesicles detected are autophagosomes, they are possibly not identical to the small vesicles detected in GFP-TIP2;1 plants or by ACAIN labeling. The presence of those small vesicles may indicate two distinct phenomena. The first one is the alteration of vacuole biogenesis similar to that shown in Arabidopsis pat mutants (Zwiewka et al. 2011). Tonoplast proteins may be present in the membrane systems of prevacuolar compartments (PVCs), when membrane traffic between PVC and vacuolar compartments is inhibited (Foresti et al. 2006). The second possibility is the enhanced lytic vacuole formation. To our knowledge, TIP3;1 and not TIP2;1 is associated to membranes of autophagosomes (Moriyasu et al. 2003). On the other hand, vesicles observed in this work are clearly delimited by tonoplasts. TIP2;1 used in our study as one of the tonoplast markers is known to be localized in both lytic and storage vacuoles (Martinoia et al. 2000).

GFP-TIP2; 1 signals showed MCY-LR-induced alterations of vacuolar organization, similar to those shown by ACAIN/ CACAIN labeling (Fig. 5a-e). MCY-LR (an inhibitor of both PP1 and PP2A) induced more pronounced vacuolar alterations than OA (inhibits preferentially PP2A) and Tm (PP1) (Fig. 6f-i). Therefore, probably both PP1 and PP2A play an 
517 important role in the differentiation of a normal vacuolar sys518 tem. It is worth mentioning that to date, there is practically no 519 information on how protein phosphatase inhibitors influence 520 plant endomembrane integrity. For vertebrate cells, it is 521 known that MCY-LR induces ER stress, mitochondrial dam522 age, and autophagy (Chen and Xie 2016). Thus, our new 523 fluorochromes ACAIN and CACAIN are presumably suitable 524 for the detection of multiple vacuole types and stress-induced 525 vacuolar changes. Taken together, the ACAIN/CACAIN la526 beling and TEM studies, the protein phosphatase inhibitor 527 MCY-LR induces probably two types of changes: (1) alter528 ation of vacuolar organization and (2) the formation of 529 autophagosome-like structures.

In conclusion, ACAIN and CACAIN can be of universal use for the preferential labeling of tonoplasts in living plant cells and the study of tonoplast dynamics. Therefore, they may be good alternatives to the use of tonoplast-specific GFP-fusion proteins widely used for this purpose. These fluorochromes are suitable for the detection of stress-related changes of vacuolar organization as revealed by treatments with protein phosphatase inhibitors. In addition, even though ACAIN labeling is not restricted to a single protein with hydrophobic motifs, it is suitable for the detection and possible further identification of proteins with cysteine-rich hydrophobic motifs, among them tonoplast-specific proteins following SDS-PAGE.

Acknowledgements This work was financially supported by the grants K-116465 and K-120638 given by NKFIH (National Research, Development and Innovation Office, Hungary) and GINOP-2.3.2-152016-00041 and GINOP-2.3.3.-15-2016-00030 project. The project is co-financed by the European Union and the European Regional Development Fund. CM was supported by the Balassi Institute/Campus Hungary Mobility Support No. B2/2H/7717 for a mobility to the University of Guelph, ON, Canada in 2014. MN was supported by the János Bolyai Research Scholarship of the Hungarian Academy of Sciences. JM acknowledges a Discovery Grant from NSERC, Canada. We would like to thank Prof. Ferenc Erdődi for providing us with okadaic acid and tautomycin and Sean Cutler for the GFP-TIP2;1 transgenic Arabidopsis line.

\section{Compliance with ethical standards}

Conflict of interest The authors declare there is no conflict of interest regarding the contents of this manuscript.

\section{References}

Allen MM (1968) Simple conditions for the growth of unicellular bluegreen algae on plates. J Phycol 4(1):1-4. https://doi.org/10.1111/j. 1529-8817.1968.tb04667.x

Batistič O (2012) Genomics and localization of the Arabidopsis DHHCcysteine-rich-domain S-acyltransferase protein family. Plant Physiol 160(3):1597-1612. https://doi.org/10.1104/pp.112.203968

Beebo A, Thomas D, Der C, Sanchez L, Leborgne-Castel N, Marty F, Schoefs B, Bouhidel K (2009) Life with and without AthTIP1;1, an
Arabidopsis aquaporin preferentially localized in the apposing tonoplasts of adjacent vacuoles. Plant Mol Biol 70(1-2):193-209. https:// doi.org/10.1007/s11103-009-9465-2

Boevink P, Oparka K, Santa Cruz S, Martin B, Betteridge A, Hawes C (1998) Stacks on tracks: the plant Golgi apparatus traffics on an actin/ER network. Plant J 15(3):441-447. https://doi.org/10.1046/j. 1365-313X.1998.00208.x

Bolte S, Talbot C, Boutte Y, Catrice O, Read ND, Satiat-Jeunemaitre B (2004) FM-dyes as experimental probes for dissecting vesicle trafficking in living plant cells. J Microsc 214(2):159-173. https://doi. org/10.1111/j.0022-2720.2004.01348.x

Bouaïcha N, Maatouk I (2004) Microcystin-LR and nodularin induce intracellular glutathione alteration, reactive oxygen species production and lipid peroxidation in primary cultured rat hepatocytes. Toxicol Lett 148(1-2):53-63. https://doi.org/10.1016/j.toxlet.2003. 12.005

Bradford MM (1976) A rapid and sensitive method for the quantitation of microgram quantities of protein utilizing the principle of protein-dye binding. Anal Biochem 72(1-2):248-254. https://doi.org/10.1016/ 0003-2697(76)90527-3

Campos A, Vasconcelos VM (2010) Molecular mechanisms of microcystin toxicity in animal cells. Intl J Mol Sci 11(1):268-287. https://doi.org/10.3390/ijms11010268

Chen L, Xie P (2016) Mechanisms of microcystin-induced cytotoxicity and apoptosis. Mini-Rev Med Chem 16(13):1018-1031. https://doi. org/10.2174/1389557516666160219130407

Cutler SR, Ehrhardt DW, Griffitts JS, Somerville CR (2000) Random GFP::cDNA fusions enable visualization of subcellular structures in cells of Arabidopsis at a high frequency. Proc Natl Acad Sci U S A 97(7):3718-3723. https://doi.org/10.1073/pnas.97.7.3718

Emans N, Zimmermann S, Fischer R (2002) Uptake of a fluorescent marker in plant cells is sensitive to brefeldin A and wortmannin. Plant Cell 14(1):71-86. https://doi.org/10.1105/tpc.010339

Feraru E, Paciorek T, Feraru MI, Zwiewka M, De Groodt R, De Rycke R, Kleine-Vehn J, Friml J (2010) The AP-3 b adaptin mediates the biogenesis and function of lytic vacuoles in Arabidopsis. Plant Cell 22(8):2812-2824. https://doi.org/10. $1105 /$ tpc. 110.075424

Foresti O, daSilva LLP, Denecke J (2006) Overexpression of the Arabidopsis syntaxin PEP12/SYP21 inhibits transport from the prevacuolar compartment to the lytic vacuole in vivo. Plant Cell 18(9):2275-2293. https://doi.org/10.1105/tpc.105.040279

Gamborg OL, Miller RA, Ojima K (1968) Nutrient requirements of suspension cultures of soybean root cells. Exp Cell Res 50(1):151-158. https://doi.org/10.1016/0014-4827(68)90403-5

Gao C, Zhuanga X, Cuia Y, Fua X, Hea Y, Zhaoa Q, Zenga Y, Shena J, Luoa M, Jiang L (2015) Dual roles of an Arabidopsis ESCRT component FREE1 in regulating vacuolar protein transport and autophagic degradation. Proc Natl Acad Sci U S A 112(6):1886-1891. https://doi.org/10.1073/pnas.1421271112

Kós P, Gorzó G, Surányi G, Borbely G (1995) Simple and efficient method for isolation and measurement of cyanobacterial hepatotoxins by plant tests (Sinapis alba L.) Anal Biochem 225(1):49-53. https://doi.org/10.1006/abio.1995.1106

Laemmli UK (1970) Cleavage of structural proteins during assembly of the head of bacteriophage T4. Nature 227(5259):680-685. https:// doi.org/10.1038/227680a0

Liu Y, Bassham DC (2012) Autophagy: pathways for self-eating in plant cells. Annu Rev Plant Biol 63(1):215-237. https://doi.org/10.1146/ annurev-arplant-042811-105441

Liu Y, Schiff M, Czimmek K, Tallóczy Z, Levine B, Dinesh-Kumar SP (2005) Autophagy regulates programmed cell death during the plant innate immune response. Cell 121(4):567-577. https://doi.org/10. 1016/j.cell.2005.03.007

Liu Y, Burgos JS, Deng Y, Srivastava R, Howell SH, Basshama DC (2012) Degradation of the endoplasmic reticulum by autophagy
570 571 572 573 574 575 576 577 578 579 580 581 582 583 584 585 586 587 588 589 590 591 592 593 594 595 596 597 598 599 600 601 602 603 604 605 606 607 608 609 610 611 612 613 614 615 616 617 618 619 620 621 622 623 624 625 626 627 628 629 630 631 632 633 634 635 
Novel fluorochromes label tonoplast in living plant cells and reveal changes in vacuolar organization after...

during endoplasmic reticulum stress in Arabidopsis. Plant Cell 42(11):4635-4651. https://doi.org/10.1105/tpc.112.101535

Maeshima M (2001) Tonoplast transporters: organization and function. Annu Rev Plant Physiol Plant Mol Biol 52:469-497

Martinoia E, Massonneau A, Frangne N (2000) Transport processes of solutes across the vacuolar membrane of higher plants. Plant Cell Physiol 41(11):1175-1186. https://doi.org/10.1093/ $\mathrm{pcp} / \mathrm{pcd} 059$

Máthé C, M-Hamvas M, Vasas G (2013) Microcystin-LR and cylindrospermopsin induced alterations in chromatin organization of plant cells. Mar Drugs 168(10):3689-3717. https://doi.org/10. 3390/md11103689

Máthé C, Beyer D, M-Hamvas M, Vasas G (2016) The effects of microcystins (cyanobacterial heptapeptides) on the eukaryotic cytoskeletal system. Mini-Rev Med Chem 16(13):1063-1077. https:// doi.org/10.2174/1389557516666160219130732

Mathur J, Mathur N, Kirik V, Kernebeck B, Srinivas BP, Hülskamp M (2003) Arabidopsis CROOKED encodes for the smallest subunit of the ARP2/3 complex and controls cell shape by region specific fine F-actin formation. Development 130(>14):3137-3146. https://doi. org/10.1242/dev.00549

Meckel T, Hurst AC, Thiel G, Homann U (2004) Endocytosis against high turgor: intact guard cells of Vicia faba constitutively endocytose fluorescently labeled plasma membrane and GFPtagged $\mathrm{K}^{+}$channel KAT1. Plant J 39(2):182-193. https://doi.org/ 10.1111/j.1365-313X.2004.02119.x

Moriyasu Y, Hattori M, Jauh GY, Rogers JC (2003) Alpha tonoplast intrinsic protein is specifically associated with vacuole membrane involved in an autophagic process. Plant Cell Physiol 44(8):795802. https://doi.org/10.1093/pcp/pcg100

Murashige T, Skoog F (1962) A revised medium for rapid growth and bioassays with tobacco tissue cultures. Physiol Plant 15(3):473-497. https://doi.org/10.1111/j.1399-3054.1962.tb08052.x

Nagy M, Rácz D, Nagy ZL, Nagy T, Fehér PP, Purgel M, Zsuga M, Kéki S (2016) An acrylated isocyanonaphthalene based solvatochromic click reagent: optical and biolabeling properties and quantum chemical modeling. Dyes Pigments 133:445-457. https://doi.org/10. 1016/j.dyepig.2016.06.036
Nelson BK, Cai X, Nebenführ A (2007) A multicolored set of in vivo organelle markers for co-localization studies in Arabidopsis and other plants. Plant J 51(6):1126-1136. https://doi.org/10.1111/j. 1365-313X.2007.03212.x

Oparka KJ (1994) Plasmolysis: new insights into an old process. New Phytol 126(4):571-591. https://doi.org/10.1111/j.1469-8137.1994. tb02952.x

Reinhardt H, Hachez C, Bienert MD, Beebo A, Swarup K, Voß U, Bouhidel K, Frigerio L, Schjoerring JK, Bennett MJ, Chaumont F (2016) Tonoplast aquaporins facilitate lateral root emergence. Plant Physiol 170:1640-1654. https://doi.org/10.1104/pp.15.01635

Schüssler MD, Alexandersson E, Bienert GP, Kichey T, Laursen KH, Johanson U, Kjellbom P, Schoellring JF, Jahn TP (2008) The effects of the loss of TIP $1 ; 1$ and TIP1;2 aquaporins in Arabidopsis thaliana. Plant J 56(5):756-767. https://doi.org/10.1111/j.1365-313X.2008. 03632.x

Swanson SJ, Bethke PC, Jones RL (1998) Barley aleurone cells contain two types of vacuoles: characterization of lytic organelles by use of fluorescent probes. Plant Cell 10(5):685-698. https://doi.org/10. $1105 /$ tpc. 10.5 .685

Swingle M, Ni L, Honkanen RE (2007) Small molecule inhibitors of Ser/ Thr protein phosphatases: specificity, use and common forms of abuse. Methods Mol Biol 365:23-38. https://doi.org/10.1385/159745-267-X:23

Vasas G, Gáspár A, Páger C, Surányi G, Máthé C, M-Hamvas M, Borbély G (2004) Analysis of cyanobacterial toxins (anatoxin-a, cylindrospermopsin, microcystin-LR) by capillary electrophoresis. Electrophoresis 25(1):108-115. https://doi.org/10.1002/elps. 200305641

Zhao J, Dixon RA (2009) MATE transporters facilitate vacuolar uptake of epicatechin 3'-O-glucoside for proanthocyanidin biosynthesis in Medicago truncatula and Arabidopsis. Plant Cell 21(8):23232340. https://doi.org/10.1105/tpc.109.067819

Zwiewka M, Feraru E, Möller B, Hwang I, Feraru MI, Kleine-Vehn J, Weijers D, Friml J (2011) The AP-3 adaptor complex is required for vacuolar function in Arabidopsis. Cell Res 21(12):1711-1722. https://doi.org/10.1038/cr.2011.99
674

675

676

677

678

679

680

681

682

683

684

685

686

687

688

689

690

691

692

693

694

695

696

697

698

699

700

701

702

703

704

705

706

707

708

709 


\section{AUTHOR QUERIES}

\section{AUTHOR PLEASE ANSWER ALL QUERIES.}

Q1. The citation "Bouaïcha and Maatuk 2004" has been changed to "Bouaïcha and Maatouk, 2004" to match the author name/date in the reference list. Please check if the change is fine in this occurrence and modify the subsequent occurrences, if necessary.

Q2. Figure 5 contains poor quality of text inside the artwork. Please do not re-use the file that we have rejected or attempt to increase its resolution and re-save. It is originally poor, therefore, increasing the resolution will not solve the quality problem. We suggest that you provide us the original format. We prefer replacement figures containing vector/editable objects rather than embedded images. Preferred file formats are eps, ai, tiff and pdf. 